\title{
Managing the difficult airway
}

\section{A survey of doctors with different seniority in China}

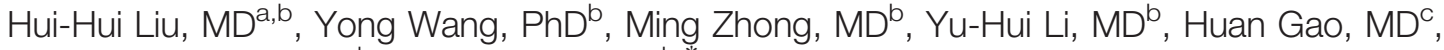 \\ Jian-Feng Zhang, MD', Wu-Hua Ma, $\mathrm{PhD}^{\mathrm{b}, *}$
}

\begin{abstract}
Although equipment and human resources are vital elements of difficult airway management (DAM). But the approach and practice of DAM are more important. So, we conducted the present survey to address anesthesiologists of different working years in this knowledge gap.

This nationwide cross-sectional study was conducted from October 27, 2016 to November 27, 2016. The survey was completed online in New Youth Anesthesia Forum including DAM assessment, anticipated and unanticipated DAM, difficult airway algorithm, use of the front of neck access (FONA) technique and training, DAM outside the operation room, and difficult extubation management.

We received 1935 replies (44\%). Mouth opening and Mallampati classification were the most common methods to evaluate difficult airways. When suffering from unanticipated difficult airway $63 \%$ less than 10 years anesthesiologists (LA) and $65 \%$ more than 10 years anesthesiologists (MA) would ask for help after trying 1 to 2 times $(P=.000)$. More than $70 \%$ of $L A$ and MA respondents reported preferring cannula cricothyrotomy to deal with emergency airway, 507 (41.6\%) MA respondents reported that they used FONA techniques to save patients' lives $(P=.000)$. Nearly $70 \%$ respondents worried full stomach when intubated outside operation room and more than $80 \%$ respondents selected auscultation to identify the placement. More than $80 \%$ respondents had not used Bougie to assist extubation. A 73.2\% respondents know ABS algorithm and $96.4 \%$ know Chinese airway expert consensus among MA respondents, this was significant to $L A$ respondents $(P=.000)$.

The respondents in the LA and MA have a training gap in their evaluation of difficult airways, trained and used FONA emergency skills, facilitated of the airway guidelines at home and abroad. Also, we should provide more airway theory and skill training to our young doctors to advanced airway skills.

Abbreviations: $\mathrm{A}=$ ask for help, $\mathrm{B}=$ breathing, $\mathrm{CICO}=$ "can't ventilate can't oxygenate," DAM = difficult airway management, $E T$ = endotracheal tube, FONA = used of front of neck access, LA = less than 10 years anesthesiologists, MA = more than 10 years anesthesiologists, $\mathrm{OR}=$ operation room, $\mathrm{RSAI}=$ rapid sequence anesthesia induction, $\mathrm{S}=\mathrm{S} 1$ spontaneous breathing, $\mathrm{S} 2=\mathrm{stab}, \mathrm{S} 3$ = surgical airway, VFIS = video flexible intubation scope.
\end{abstract}

Keywords: different seniority doctors, difficult airway, extubation, intubation, survey, training

\section{Editor: Bernhard Schaller.}

HHL and YW contributed equally to this work.

The approach and practice of difficult airway management address anesthesiologists who are of different working years in China.

Following approval from the Institutional Review Board of the First Affiliated Hospital of Guangzhou University of Chinese Medicine (Guangzhou, China) (No. ZYYEC-ERK [2019]141). Every anesthesiologist's member in the New Youth Anesthesia Forum participates in the survey invitation anonymously by WeChat. Before the investigation, they would first choose whether to agree with the contents of the investigation. The data we collect were all part of the agreement.

Additional file 1: 2016 National Difficult Airway Management Status Survey. Includes the 63-question survey used for the needs-assessment study. (DOCX 41 kb) This study was funded by the Guangzhou University of Traditional Chinese Medicine High-level Construction Project (A1-AFD018171Z11075), Outstanding Youth Fund of the "Innovative Strong Institute" (10900088), and Guangzhou Science and Technology Plan Project (201707010297). These funding sources had no role in the design of this study, they separately performed data execution, results analysis, and statistics in this study.

The authors have no conflicts of interest to disclose.

The datasets generated during and/or analyzed during the present study are publicly available.

${ }^{a}$ Department of Anesthesiology, The First Clinical Medical College of Guangzhou University of Chinese Medicine, Guangzhou, Guangdong, P.R. China, ${ }^{b}$ Department of Anesthesiology, The First Affiliated Hospital of Guangzhou University of Chinese Medicine, Guangzhou, Guangdong, P.R. China, ${ }^{\circ}$ Department of Anesthesiology, Fangcheng General Hospital, Nanyang, Henan, P.R. China, ${ }^{d}$ Department of Anesthesiology, The Affiliated Hospital of Hubei University of Arts and Science of Xiangyang Central Hospital, Xiangyang, Hubei, P.R. China.

* Correspondence: Wu-Hua Ma, Department of Anesthesiology, The First Affiliated Hospital of Guangzhou University of Chinese Medicine, 16 Airport Road, Guangzhou, Guangdong 510405, P.R. China (e-mail: gzymwhqd@163.com)

Copyright $\odot 2021$ the Author(s). Published by Wolters Kluwer Health, Inc.

This is an open access article distributed under the terms of the Creative Commons Attribution-Non Commercial License 4.0 (CCBY-NC), where it is permissible to download, share, remix, transform, and buildup the work provided it is properly cited. The work cannot be used commercially without permission from the journal.

How to cite this article: Liu HH, Wang Y, Zhong M, Li YH, Gao H, Zhang JF, Ma WH. Managing the difficult airway: a survey of doctors with different seniority in China. Medicine 2021;100:38(e27181).

Received: 27 February 2020 / Received in final form: 16 August 2021 / Accepted: 23 August 2021

http://dx.doi.org/10.1097/MD.0000000000027181 


\section{Introduction}

Airway management out/in the operation room $(\mathrm{OR})$ is one of the most vital initial steps of resuscitation and is also a critical skill requiring mastery by an anesthesiologist. The rate of difficult endotracheal intubation in elective surgery is $0.5 \%$ to $8.5 \%,{ }^{[1-2]}$ outside OR ranges from $6.1 \%$ to $23.5 \% .{ }^{[3-4]}$ Maybe because of the more critical patient population, the lesser controlled setting, and the inadequate opportunity for a complete evaluation of the patient. ${ }^{[5]}$ Failure to obtain and maintain adequate airway oxygenation and ventilation will result in pathological damage that is not reversed, such as hypoxia, aspiration, and cardiac arrest. To avoid many uncommon, yet critical failures, many tools for airway management have been developed, such as the laryngeal mask airway and video tube, which have been demonstrated efficacious and easily mastered by anesthesia in many settings. ${ }^{[6-7]}$ But, when we encounter the life-threatening “can't ventilate can't oxygenate" (CVCO) scenario, the approach and practice of doctors of difficult airway management (DAM) are more important. As we know that this will take some time to train, that may be differences between doctors with different years of work.

Instruction in airway management during anesthesiology residency was surveyed by Hagberg et $\mathrm{al}^{[8]}$ in 2003. In 2011, the United States and Canada ${ }^{[9]}$ re-surveyed assumed that there has been a convert in the education of anesthesia residents in airway management regarding choices of airway devices and modes of training. However, the approach and practice of DAM between anesthesiologists who are of different working years in China are unknown. We also believe that if a doctor works for 10 years, his thinking mode of difficult airway algorithm has been basically settled. So, this survey takes the length of working time (10 years) as a boundary address to knowledge the gaps.

\section{Methods}

A survey was designed by the authors and discussed with our local Airway Management Society, who confirmed that formal approval by an Ethics Committee was not required.

\subsection{Study design}

The survey was completed online from October 27, 2016 to November 27, 2016 at the New Youth Anesthesia Forum, with more than 78,000 registered anesthesia experts. A survey was sent to all anesthesiologist members by WeChat. Each IP address was allowed to complete the survey once. The respondents can choose a computer or mobile device to complete the survey.

\subsection{Survey items}

A questionnaire, which was based on the clinical experience of Dr $\mathrm{Ma}$. It also referred to some relevant studies conducted in other countries which have similarly assessed emergency departments, ${ }^{[10-11]}$ intensive care units, ${ }^{[12-13]}$ ORs, ${ }^{[14-15]}$ and prehospital settings. ${ }^{[16-17]}$ We then distributed the drafts to the survey team members. It was completed in August 2016. The DAM included specifically in the survey were as follows:

Survey items covered 9 areas:

(i) Airway assessment

(ii) Anticipated DAM

(iii) Anticipated difficult airway tools selection (iv) Unanticipated DAM

(v) DAM outside the operating room

(vi) Recognition of DAM algorithm

(vii) Front of neck access technique

(viii) Difficult airway extubation

(ix) Difficult airway training

The survey questions included basic demographic information about the anesthesiologist's hospital level (grade I, II, or III). To minimize the bias for several open issues, question formats were used: "Yes" or "No" boxes, choose the best possible answer from 2 to 5 alternatives options. No monetary compensation was provided for any form of participation in this survey.

\section{Statistical analysis}

All survey items were evaluated using descriptive statistics. The associations between the results were analyzed using a Fisher exact test which included only the complete data sets. Nonparametric tests were used for continuous variables. All statistical analyses were performed using IBM SPSS Statistics for Windows, version 20 (IBM Corp., Armonk, NY). All tests were 2 tailed with a type I error rate of 0.05 .

\section{Results}

The survey was conducted from October 27, 2016 to November 27,2016 . The server can record how many members open and read the invitation. The study included only those members who read the survey invitation.

A total of 4437 members read invitations. In total 2478 forms were returned, the remaining (at least partially completed) 1935 questionnaires were analyzed and the overall response rate was $44 \%$. Responses were received from hospitals in 31 provinces of China. The distribution of working years was: $>10$ years $63 \%$ $(1222 / 1935)$ and $<10$ years $37 \%(713 / 1935)$.

\subsection{Evaluation of difficult airway}

Table 1 shows responses by working years (of respondents) to the questions concerning evaluation of difficult airway. All groups of respondents believed that Mouth Opening and Mallampati Classification are the most commonly used methods. The number of thyromental distance and atlanto-occipital joint extension selected medium. Approximately 839/1221 of the respondents ( $>10$ years) think there are other ways to evaluate, but less than 10 years anesthesiologists (LA) only 51/713 $(P=.000)$.

\section{Table 1}

The index of LA and MA to evaluate the difficult airway (multiple selections).

\begin{tabular}{lccc}
\hline & $\begin{array}{c}<\mathbf{1 0} \text { years } \\
\mathbf{N}=\mathbf{7 1 3}\end{array}$ & $\begin{array}{c}>\text { 10 years } \\
\mathbf{N}=\mathbf{1 2 2 1}\end{array}$ & $\boldsymbol{P}$ \\
\hline Mouth opening & 637 & 1044 & .016 \\
Thyromental distance & 518 & 242 & .000 \\
Atlanto-occipital joint extension & 338 & 507 & .012 \\
Mallampati classification & 544 & 527 & .000 \\
Cormack-Lehane classification & 79 & 474 & .000 \\
Others & 51 & 839 & .000
\end{tabular}

$L A=$ less than 10 years anesthesiologists, $M A=$ more than 10 years anesthesiologists. 


\section{Table 2}

The approach to deal with an anticipated difficult airway.

\begin{tabular}{lccc}
\hline & $<\mathbf{1 0 \text { years }}$ & $>\mathbf{1 0 \text { years }}$ & \\
& $\mathbf{N}=\mathbf{7 1 3}$ & $\mathbf{N = 1 2 2 1}$ & $\boldsymbol{P}$ \\
\hline TA+ awake VFIS & $63(8.8 \%)$ & $112(9.2 \%)$ & .000 \\
TA+ sedation + awake VFIS & $426(59.7 \%)$ & $888(72.7 \%)$ & .000 \\
Sedation + analgesia + VFIS & $63(8.8 \%)$ & $56(4.6 \%)$ & .000 \\
RSAl & $161(22.6 \%)$ & $165(13.5 \%)$ & .000 \\
\hline
\end{tabular}

RSAI = rapid sequence anesthesia induction, $T A=$ topical anesthesia, VFIS = video flexible intubation scope.

\subsection{Anticipated-difficult airway and tools}

Table 2 indicates how to deal with anticipated difficult airway between LA and more than 10 years anesthesiologists (MA) of $59.7 \%$ respondents who described elective awake video flexible intubation scope (VFIS) intubation combined topical anesthesia (TA) and sedation to deal with anticipated difficult airway among LA, but $72.7 \%$ respondents among MA. Fewer than one-third reported elective use rapid sequence anesthesia induction. The number of VFIS intubation combined sedation and VFIS intubation combined both TA performed by the respondents was small but greater than zero.

Figure 1 shows how to select the airway tool for each respondent. A total of 310 of 713 possible LA respondents reported videolaryngoscope of selection. Two hundred twenty of a possible 713 LA respondents described selective use of VFIS intubation. A $12.9 \%$ reported regular use of Macintosh laryngoscope, and less than $15 \%$ reported to chose optical stylets, lightwand, or intubating laryngeal mask.

The trend is the same among MA respondents, and there was no statistical difference between groups $(P=.263)$. They tend to choose visualization and easy to performed tools.

\subsection{Unanticipated difficult airway}

Not all difficult airways can be evaluated in advance. When we are in a life-threatening difficult intubation or even CVCI scenario, what should we do first. A $63 \%$ LA and $65 \%$ MA would ask for help after trying 1 to 2 times. A $23 \%$ LA may

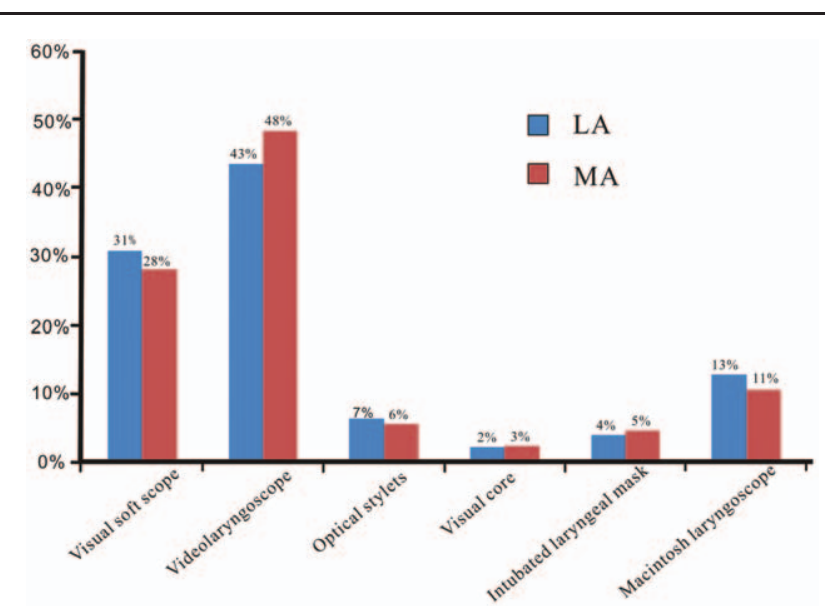

Figure 1. Proportion of $L A$ and MA preferred intubation tools for patients with an anticipated difficult airway. $L A=$ less than 10 years anesthesiologists, $M A=$ more than 10 years anesthesiologists.

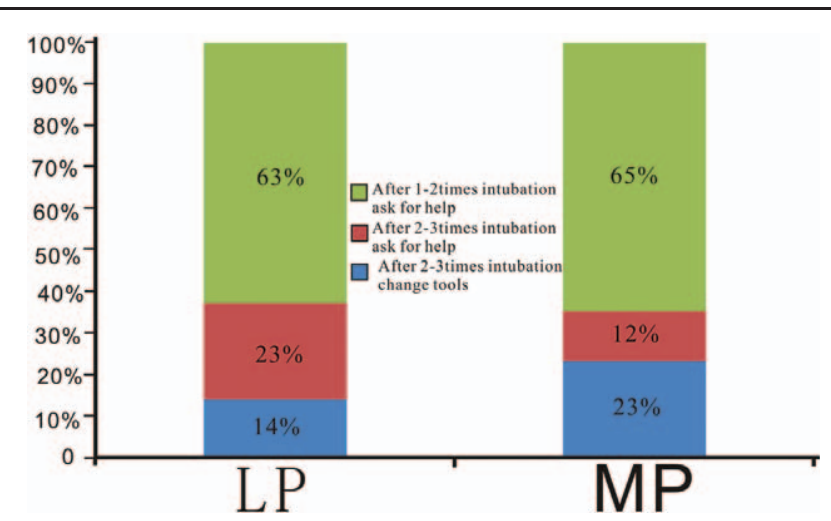

Figure 2. Proportion of $L A$ and MA practice unanticipated difficult airway. $L A=$ less than 10 years anesthesiologists, MA=more than 10 years anesthesiologists.

choice try 2 to 3 times before seeking help, while 23\% MA selected change intubation tools after trying 2 to 3 times (Fig. 2) $(P=.000)$.

\subsection{Front of neck access emergency technique}

Table 3 and Fig. 3 indicates when we suffered the life-threatening can't ventilate can't oxygenate (CICO) scenario, use of the front of neck access (FONA) emergency techniques like cannula cricothyrotomy, surgical cricothyrotomy, and tracheotomy's performed and attitudes to it between LA and MA respondents, and this was significant $(P=.000)$.

More than $70 \%$ of LA respondents reported preferring cannula cricothyrotomy to deal with CICO, but only 47 $(6.9 \%)$ used it by themselves. The number of surgical cricothyrotomy and tracheotomy performed by LA respondents was small but greater than zero. Among LA, less than one-third respondents reported used the FONA emergency technique. While among MA, 507 respondents $(41.6 \%)$ reported that they used FONA emergency techniques to save patients' lives. Nearly 931 respondents $(76.4 \%)$ preferred cannula cricothyrotomy like LA respondents. The number of surgical cricothyrotomy, tracheotomy, and other FONA emergency techniques performed by MA respondents was small but greater than zero. Two hundred twenty-nine MA respondents $(18.8 \%)$ responded that they had used the cannula cricothyrotomy technique by themselves. Whereas a fraction of programs had operated surgical cricothyrotomy $(6.0 \%)$ and tracheotomy $(4.2 \%)$.

\section{Table 3}

First choice of FONA emergency technique when suffered $\mathrm{CICO}$ scenario.

\begin{tabular}{lcc}
\hline & $<\mathbf{1 0}$ years $\mathbf{N}=\mathbf{7 1 3}$ & $>\mathbf{1 0}$ years $\mathbf{N}=\mathbf{1 2 2 1}$ \\
\hline Cannula cricothyrotomy & $506(71.0 \%)$ & $931(76.2 \%)$ \\
Surgical cricothyrotomy & $56(7.9 \%)$ & $71(5.8 \%)$ \\
Tracheotomy & $90(12.6 \%)$ & $107(8.7 \%)$ \\
Others & $61(7.6 \%)$ & $109(8.9 \%)$ \\
\hline
\end{tabular}

The first choice of FONA emergency technique significantly different between LA and MA responders $P=.000$.

$\mathrm{CICO}=$ can't ventilate can't oxygenate, FONA $=$ front of neck access, $L A=$ less than 10 years anesthesiologists, $M A=$ more than 10 years anesthesiologists. 




Figure 3. The proportion of anesthetists used of the front of neck access emergency technique. $L A=$ less than 10 years anesthesiologists, $M A=m o r e$ than 10 years anesthesiologists, FONA = front of neck access.

\subsection{DAM outside the operating room}

Table 4 details the responses of worries when intubated outside OR: including full stomach, difficult airway, environmental impact, or others. A 484 of 713 possible LA respondents preferred a worried full stomach. Among MA respondents even high to $71 \%$. The number of difficult airway and environmental performed by MA and LA respondents was small but greater than zero.

How to identify the ETT placement outside of OR is very important. More than $80 \%$ respondents selected auscultation. Despite the high availability of capnometry, its routine use for endotracheal intubation was reported by $6.5 \%$ of the LA and $5.5 \%$ of the MA outside of OR. Other minority of respondents selected chest rise. The number of VFIS and other methods, such as tube fogging, direct visualization performed by MA and LA respondents was small but greater than zero.

\subsection{Difficult airway extubation}

Fig. 4 indicates removal of the endotracheal tube in a patient with difficult airway. A 512 of 713 LA respondents experienced or assisted difficult airway re-intubation. Among them, $12.8 \%$ of

\section{Table 4}

The most worried condition when we intubated out of the OR and identified the placement of the ETT.

\begin{tabular}{lcc}
\hline & $\begin{array}{c}<\mathbf{1 0 y e a r s} \\
\mathbf{N}=\mathbf{7 1 3}\end{array}$ & $\begin{array}{c}>\mathbf{1 0} \text { years } \\
\mathbf{N}=\mathbf{1 2 2 1}\end{array}$ \\
\hline The most worried & & \\
$\quad$ Full stomach & $484(67.9 \%)$ & $867(71.0 \%)$ \\
$\quad$ Difficult airway & $61(8.6 \%)$ & $66(7.0 \%)$ \\
$\quad$ Environmental impact (family members) & $1(0.1 \%)$ & $4(0.3 \%)$ \\
$\quad$ Others & $167(23.4 \%)$ & $264(21.6 \%)$ \\
How to identify the placement of the ETT & & \\
$\quad$ Auscultation & $581(81.5 \%)$ & $1003(82.1 \%)$ \\
Chest rise & $40(5.6 \%)$ & $105(8.6 \%)$ \\
Capnography & $46(6.5 \%)$ & $67(5.5 \%)$ \\
Graphics of VIFS & $35(4.9 \%)$ & $31(2.5 \%)$ \\
$\quad$ Others & $11(1.5 \%)$ & $13(1.1 \%)$ \\
\hline
\end{tabular}

The most worried when intubated outside $\mathrm{OR}$ significantly no different $P=.451$. The method respondents selected to identify trachea significantly different $P=.006$.

$\mathrm{ETT}=$ endotracheal tube, $\mathrm{OR}=$ operation room, $\mathrm{VFIS}=$ video flexible intubation scope. the patients failed re-intubation and were faced with the use of FONA emergency technical to rescue the patients. Nearly $86 \%$ of the LA and $80.1 \%$ of the MA had not used Bougie to assist in the extubation of difficult airways. So, even among MA, $84.5 \%$ respondents experienced or assisted difficult airway re-intubation, and $20.2 \%$ failed it.

\subsection{Airway management training}

To clarify the current situation and to provide a reference point, this requested information on the airway management training programs available in each doctor, including difficult airway treatment algorithms at home and abroad, airway management training, such as surgical cricothyrotomy, tracheotomy, retrograde intubation, and transtracheal jet ventilation.

A $41.1 \%$ LA respondents had already attended an airway training course, while $158(22.2 \%)$ intended cannula cricothyrotomy and transtracheal jet ventilation, only $97(13.6 \%)$ respondents intended surgical cricothyrotomy and $95(13.2 \%)$ to the retrograde intubation training. With the increase of working years, 692 of a possible $1221 \mathrm{MA}$ respondents had already attended an airway training course, 422(34.6\%) intended cannula cricothyrotomy and transtracheal jet ventilation, $304(24.9 \%)$ respondents intended surgical cricothyrotomy, and $231(18.9 \%)$ to the retrograde intubation training (Fig. 5).

Fig. 6 shows the understanding of difficult airway algorithms at home and in America. Respondents who never read the American Society of Anaesthesiologists algorithm occupied the largest proportion. While only a minority of respondents did not know the Chinese airway expert consensus and DAM ABS algorithm. Among MA, even $73.2 \%$ respondents know ABS algorithm and 96.4\% know Chinese airway expert consensus.

\section{Discussion}

The New Youth Anesthesia Forum has more than 78,000 registered anesthesiologists. New Youth Anesthesia WeChat public number can calculate the number of people who read the survey invitation and record their locations. Therefore, we can calculate the response rate. Jane Candlish suggested that the minimum number of survey answers required for a survey to be effective should be equal to the number of questions times 10 . $^{[18]}$ In this study, we received the 1935 reply. The location of the 
MA

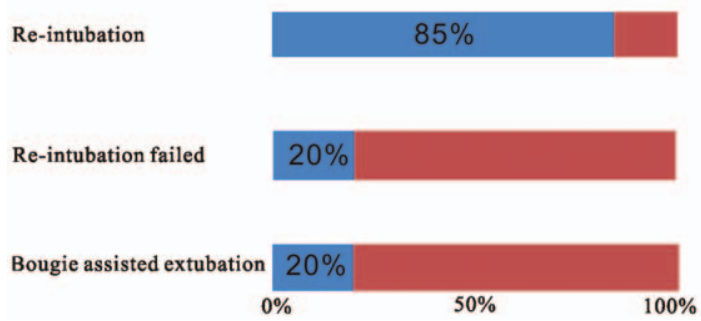

LA

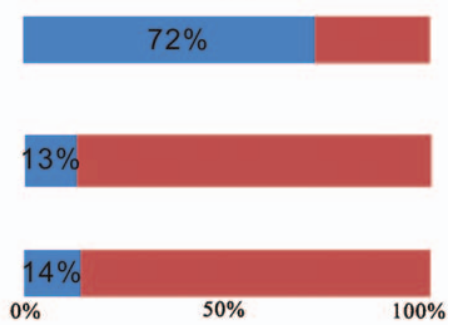

Figure 4. The proportion of $L A$ and MA deal with difficult airway extubation. $L A=$ less than 10 years anesthesiologists, MA=more than 10 years anesthesiologists.

respondents indicated that the participants came from every province in China.

This survey provides the differences of LA and MA evaluation, selection of tools, difficult airway training, management of difficult airway in China. Airway management has always been a cornerstone of anesthetic practice. ${ }^{[19]}$ Although large training centers may have many resources available, many anesthetists practice in settings where these resources are limited or nonexistent. In many settings, the anesthetist is the sole member of the airway team throughout the hospital in China. So, we conducted the present survey to address anesthesiologists of different working years in this knowledge gap in China.

Our study demonstrates that most LA and MA selected mouth opening as the first index in the evaluation of difficult airway, therefore the second choice among MA was experiential methodologies, and LA selected less, they tend to Mallampati classification which was classical method in the book (Table 1). Although the assessment of DAM is different, they are basically consistent in the treatment of anticipated difficult airways (Table 2). A $426(60 \%)$ LA and $888(73 \%)$ MA put TA+ sedation + VFIS as the first choice to manage anticipated DAM. Cricothyrotomy can provide airway anesthesia for an awake VFIS, it can also provide a valuable experience in the lifethreatening CICO scenario. This is also consistent with the ASA guidelines $^{[20]}$ recommendation.

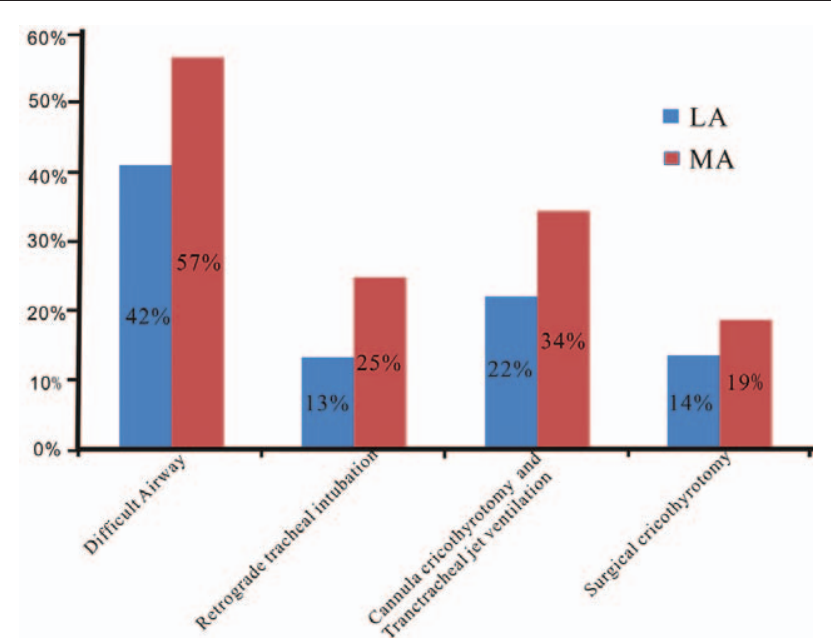

Figure 5. Proportion of $L A$ and MA received airway management training. $L A=$ less than 10 years anesthesiologists, $M A=$ more than 10 years anesthesiologists.
Kim et al ${ }^{[21]}$ suggested that there was no failed attempt when the resident had performed more than 30 times training endoscopies. K Latif ${ }^{[22]}$ recommends 10 fiberoptic intubations on asleep patients and 15 to 20 on awake patients for acceptable expertise. So adequate training in DAM can enhance our confidence.

As mentioned above, the anesthetist is the sole member of the airway team throughout the hospital in China. Out of OR, what respondents are most worried about is the patients with full stomach (68\% LA and 71\% MA) (Table 4). Maybe they are also worried about some other situations, such as bleeding, drinking, and so on. They care about environmental impactless. After intubation, more than $80 \%$ respondents selected auscultation which is considered to be third-class evidence to verify the placement of ETT (Table 4) outside of the OR. Less than $15 \%$ of the respondents routinely used capnography (second-class evidence) and chest rise (third-class evidence) for ETT placement verification. They rarely trust the intubation graphics (first-class evidence) of the video laryngoscope including VFIS (gold evidence) alone. The results showed that the increased use of experience to verify ETT placement, may be because of lacked theoretical knowledge. We can provide more training on this in the future. If there are no video tools for ETT placement. Zamani et $\mathrm{al}^{[23]}$ showed that the increased use of $\mathrm{CO}_{2}$ monitors was the single change that has the greatest potential to prevent death from airway complications outside the OR.

When we encounter difficult airways, we should choose the tools we are most familiar with. Approximately half of the LA and MA put videolaryngoscope as the first choice and VFIS intubations as the second choice (Fig. 1). There are numerous benefits of videolaryngoscopy, and these include improved laryngeal view, high rates of successful rescue after the failure of direct laryngoscopy, ${ }^{[17]}$ improved training of novices, ${ }^{[24]}$ T. M. Cook ${ }^{[25]}$ suggested that videolaryngoscopy was used in $91 \%$ of operating theatres, $50 \%$ of intensive care units and obstetric theatres. Not all difficult airways can be evaluated in advance. When we are in the life-threatening difficult intubation or even CICO scenario, what should we do first. A 63\% LA and 65\% MA would ask for help after trying 1 to 2 times. A $23 \%$ LA may choice try 2 to 3 times before seeking help, while $23 \%$ MA selected change intubation tools after trying 2 to 3 times (Fig. 2). But what I want to remind is to put "ask for help" in the first place and try to maintain oxygenation, so as to reduce the risk of patients.

The incidence of can't ventilate can't intubation is rare, but when we threatened by it, 506 (71.0\%) LA and 931 (76.2\%) MA selected cannula cricothyrotomy (Table 3), 90 (12.6\%) LA and 107 (8.7\%) MA selected tracheotomy. Only very small numbers of anesthetists selected surgical cricothyrotomy. In a study 

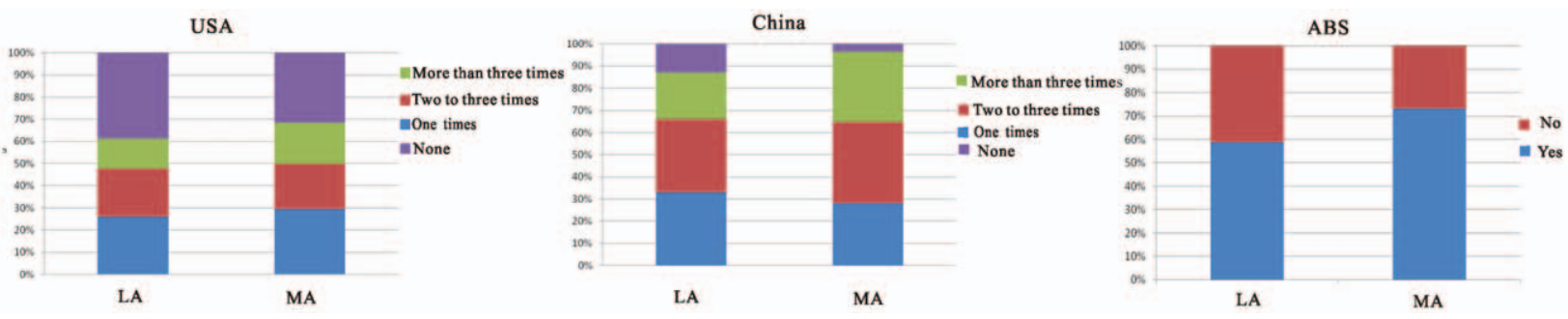

Figure 6. Proportion of $L A$ and MA who understood of airway management algorithm at home and in America. $L A=$ less than 10 years anesthesiologists, MA $=$ more than 10 years anesthesiologists, ABS: $A=$ ask for help, $B=$ breathing, $S=S 1$ spontaneous breathing, S2 stab, S3 surgical airway (See Appendix).

conducted the next year ${ }^{[28]}$ only $10 \%$ had previous experience of surgical cricothyrotomy in patients, while Hung ${ }^{[27]}$ reported that $86 \%$ of Canadian training teach surgical cricothyrotomy. Years ago, guidelines ${ }^{[26]}$ highlight the role of surgical cricothyrotomy in an emergency, which is a difficult airway skill where a training gap may exist. While cannula cricothyrotomy kits can be quickly mastered.

Though it is important to master the intubation skills, it rather has correct treatment approach. There are difficult airway treatment guidelines at home and abroad. Professor Ma also has his own set of ABS algorithm. Such as guidelines in the USA, less than $30 \%$ respondents read 1 time, most of them even do not know about it. Approximately more than one-third respondents read Chinese DAM guidelines, nearly $67 \%$ read 2 to 3 times even more than 3 times in MA and $54 \%$ in LA (Fig. 6). Among respondents, more than $60 \% \mathrm{LA}$ and $\mathrm{MA}$ read the ABS algorithm, which may be because of its simplicity, safety, and easy to remember (Fig. 6). Everyone of us may be familiar with this algorithm, as the algorithm is like a light that allows us to make the right selection in the emergency of airway treatment.

As surveyed in our paper, very few people can use the FONA emergency technique, however, cricothyroid puncture used most (Fig. 3). Among MA, they received more airway training than LA, especially DAM (57\%), cricothyroid puncture, and tracheal jet ventilation $(34 \%)$. Wong et a ${ }^{[28]}$ suggested 5 cannula cricothyrotomy on models as the minimum training requirement, but how this infer to clinical practice is unclear. Surgical cricothyrotomy was trained least. Skills can be taught on commercial mannequins or self-contained models, ${ }^{[29]}$ such as tracheotomy of isolated pig trachea and so on, although they do not really represent clinical practice.

Finally, this study surveyed difficult extubation. Every difficult airway patient is a difficult extubation object. A $72 \%$ LA and $85 \%$ MA experienced difficult extubation, and 13\% LA and $20 \%$ MA even experienced re-intubation or failed re-intubation. So we may master extubation skills to deal with it. Bougie is a good tool for handling difficult extubation. A survey of British anesthetic departments published in 2009 showed that the bougie was available on more than $90 \%$ of difficult airway trolleys. ${ }^{[0]}$ While in our survey less than $20 \%$ respondents used it (Fig. 4). That may be the direction of trainning. Meanwhile, every doctor should attend 1 or more trainings per year.

The survey summarised the current status and characteristics of Chinese DAM, compared differences in DAM between different seniority in China. The survey revealed that young doctors lagged behind in experience and theoretical knowledge in dealing with difficult airways than MA.

The important problem highlighted by the survey is that young doctors lack adequate theoretical and practical training, which implies: (1) that more attention should be paid to physician training and teaching and (2) the public awareness of DAM in China needs to be improved. Encourage schools to do more exercises on models and animals difficult airway handling.

\section{Conclusion}

The respondents in the LA and MA have a training gap in their evaluation of difficult airways, trained, and used FONA emergency skills, facilitated the airway guidelines at home and abroad. However, they are basically the same when they deal with CICV and chose tools for DAM. Any time we may put "Ask For Help" first and try to ensure the patient's "oxygenation." Also, we should provide more airway theory and skills training to our young doctors to advanced airway skills in the model, animal, and homemade tools.

\section{Limitations}

This survey has a number of limitations. First, because it is a survey, the data are self-reported, so the reporters may wish to reflect more of what the reporters want than the actual situation. Second, we can shorten the interval between respondents. Third, the survey may add images or other investigating modalities to confirm the anticipation. Fourth, we may add more DA parameters assessment criteria and DA history in the survey. Finally, we can make the choices a little bit more detailed for respondents.

\section{Acknowledgments}

Thank you very much for the help provided by the New Youth Anesthesia Forum.

\section{Author contributions}

All authors contributed to the construction of the questionnaire. HG and ZJ supervised the conductance of the survey and data collection. HL managed the data, constructed the database, and drafted the initial manuscript. WY, MZ, and YL participated in the survey design. HL and WM contributed substantially to its revision. All authors read and approved the final version of the manuscript.

Conceptualization: Wu-Hua Ma.

Data curation: Yong Wang.

Formal analysis: Ming Zhong, Wu-Hua Ma.

Methodology: Jian-Feng Zhang.

Software: Huan Gao.

Supervision: Yu-Hui Li. 
Writing - review \& editing: Hui-Hui Liu, Wu-Hua Ma.

\section{References}

[1] Norskov AK, Rosenstock CV, Wetterslev J, Astrup G, Afshari A, Lundstrom LH. Diagnostic accuracy of anaesthesiologists' prediction of difficult airway management in daily clinical practice: a cohort study of 188064 patients registered in the Danish Anaesthesia Database. Anaesthesia 2015;70:272-81.

[2] Langeron O, Cuvillon P, Ibanez-Esteve C, Lenfant F, Riou B, Le Manach $Y$. Prediction of difficult tracheal intubation: time for a paradigm change. Anesthesiology 2012;117:1223-33.

[3] Hasegawa K, Shigemitsu K, Hagiwara Y, et al. Association between repeated intubation attempts and adverse events in emergency departments: an analysis of a multicenter prospective observational study. Ann Emerg Med 2012;60:749-54.

[4] Martin LD, Mhyre JM, Shanks AM, Tremper KK, Kheterpal S. 3,423 Emergency tracheal intubations at a university hospital: airway outcomes and complications. Anesthesiology 2011;114:42-8.

[5] Michael Bernhard, Sönke Nils Bax, Thomas Hartwig, et al. Airway Management in the Emergency Department (The OcEAN-Study) - a prospective single centre observational cohort study. Scand J Trauma Resusc Emerg Med 2019;27:20.

[6] Butler KH, Clyne B. Management of the difficult airway: alternative airway techniques and adjuncts. Emerg Med Clin North Am 2003; 21:259-89.

[7] Driver BE, Scharber SK, Horton GB, Braude DA, Simpson NS, Reardon RF. Emergency department management of out-of-hospital laryngeal tubes. Ann Emerg Med 2019;7:1-9.

[8] Hagberg CA, Greger J, Chelly JE, Saad-Eddin HE. Instruction of airway management skills during anesthesiology residency training. J Clin Anesth 2003;15:149-53.

[9] Pott LM, Randel GI, Straker T, Becker KD, Cooper RM. A survey of airway training among U.S. and Canadian Anesthesiology residency programs. J Clin Anesth 2011;23:15-26.

[10] Swaminathan AK, Berkowitz R, Baker A, Spyres M. A survey to compare the utilization of video laryngoscopy devices in emergency medicine residency programs and community emergency departments. J Emerg Med 2015;48:613-9.

[11] Browne A. A lack of anaesthetic clinical attachments for emergency medicine advanced trainees in New Zealand: perceptions of directors of emergency medicine training. N Z Med J 2015;128:45-9.

[12] Haviv Y, Ezri T, Boaz M, Ivry S, Gurkan Y, Izakson A. Airway management practices in adult intensive care units in Israel: a national survey. J Clin Monit Comput 2012;26:415-21.

[13] Foy KE, Mew E, Cook TM, et al. Paediatric intensive care and neonatal intensive care airway management in the United Kingdom: the PIC-NIC survey. Anaesthesia 2018;74:114-27.

[14] Alakeson N, Flett T, Hunt V, et al. Difficult airway equipment: a survey of standards across metropolitan Perth. Anaesth Intensive Care 2014; 42:657-64.
[15] Cook TM, Kelly FE. A national survey of videolaryngoscopy in theUnited Kingdom. Br J Anesth 2017;118:593-600.

[16] Le Parc JM, Bischof JJ, King AM, et al. A randomized comparison of inhospital rescuer positions for endotracheal intubation in a difficult airway. West J Emerg Med 2018;19:660-7.

[17] Rhode MG, Vandborg MP, Bladt V, Rognås L. Video laryngoscopy in pre-hospital critical care - a quality improvement study. Scand J Trauma Resusc Emerg Med 2016;24:84.

[18] Candlish J, Dawn Teare M, Dimairo M, Flight L, Mandefield L, Walters SJ. Appropriate statistical methods for analysing partially nested randomised controlled trials with continuous outcomes: a simulation study. BMC Med Res Methodol 2018;18:1-17.

[19] Karlik J, Aziz M. Recent trends in airway management. F1000Res 2017;6:159.

[20] Caplan RA, Benumof JL, Berry FA, et al. Practice guidelines for the management of the difficult airway: an updated report by the American Society of Anesthesiologists Task Force on Management of the Difficult Airway. Anesthesiology 2013;118:251-70.

[21] Kim H, So E, Karm MH, Kim HJ, Seo KS. Learning fiberoptic intubation for awake nasotracheal intubation. J Dent Anesth Pain Med 2017;17: 297-305.

[22] K Latif R, Bautista A, Duan X, et al. Teaching basic fiberoptic intubation skills in a simulator: initial learning and skills decay. J Anesth 2016; 30:12-9.

[23] Zamani M, Esfahani MN, Joumaa I, Heydari F. Accuracy of real-time intratracheal bedside ultrasonography and waveform capnography for confirmation of intubation in multiple trauma patients. Adv Biomed Res 2018;7:95

[24] Sakles JC, Mosier J, Patanwala AE, Dicken J. Learning curves for direct laryngoscopy and GlideScopeVR video laryngoscopy in an emergency medicine residency. West J Emerg Med 2014;15:930-7.

[25] Grundgeiger T, Roewer N, Grundgeiger J, Hurtienne J, Happel O. Body posture during simulated tracheal intubation:GlideScopeVR videolaryngoscopy vs Macintosh direct laryngoscopy for novices and experts. Anaesthesia 2015;70:1375-81.

[26] Wong DT, Prabhu AJ, Coloma M, Imasogie N, Chung FF. What is the minimum training required for successful cricothyroidotmy? Anesthesiology 2003;98:349-53.

[27] Hung O. Airway management: the good the bad and the ugly. Can J Anaesth 2002;49:767-71.

[28] Henderson JJ, Popat MT, Latto IP, Pearce AC. Difficult Airway Society guidelines for management of the unanticipated difficult intubation. Anaesthesia 2004;59:675-94.

[29] Takayesu JK, Peak D, Stearns D. Cadaver-based training is superior to simulation training for cricothyrotomy and tube thoracostomy. Intern Emerg Med 2016;12:99-102.

[30] Driver BE, Prekker ME, Klien LR, et al. Effect of use a bougie vs endotracheal tube and stylet on first attempt intubation success among patients with difficult airway undergoing emergency intubation a randomized clinical trail. JAMA 2018;12:E1-1.

[31] Wu-Hua Ma. The ABS Algorithm and Practice of Difficult Airway Management. 2016;People's Health Publishing Company Inc, 